\title{
Rural Heritage: Value, Conservation and Revitalisation-From the Perspective of the Human-Land Relationship
}

\author{
Xiaofan Du and Ding Shi* \\ Department of Cultural Heritage and Museology, Fudan University, Shanghai, China \\ *Corresponding author: shidingdd@fudan.edu.cn
}

\begin{abstract}
Today, rural revitalisation has become a national issue for China, and it is one that many countries also have to face. However, a rural area is not some wilderness that is open to any type of development. The long history of agricultural civilisation has left an extensive, precious heritage in rural areas; thus, revitalising rural areas has to be based on conserving that heritage. This special issue adopts the theme of 'rural heritage': we provide a platform for dialogue between China and overseas scholars with the focus on conserving and revitalising rural heritage. There are six contributions in this special issue on matters that affect rural heritage in various countries. Among them, it is possible to understand methods of conservation design with respect to Japan's rural heritage and follow the role of rural tourism in France. It is also possible to grasp the importance of promoting a building culture in rural parts of Germany. All these contributions offer profound insights for conserving and revitalising China's rural heritage. This special issue also presents the thoughts of Chinese and foreign experts on recent issues related to China's rural heritage; it provides a comprehensive introduction to the rural heritage protection system in China. Rural heritage is a complex system. Accordingly, the topics addressed in this special issue reflect the various characteristics involved. However, if readers study these articles from the perspective of the human-land relationship, they will be able to identify the deep correlations among them.
\end{abstract}

KEYWORDS rural heritage, value, conservation, revitalisation, sustainable development, cultural landscape, human-land relationship

Today, rural revitalisation has become a national issue in China, and many experts from China and abroad are active in areas related to such revitalisation. In April 2019, the Second International Conference on Built Heritage Studies (BHS 2019), under the theme 'Built Heritage Conservation in Rural Vitalisation,' was held at Tongji University ${ }^{1}$. At the conference, experts from China and other countries discussed their experience and theoretical considerations related to rural protection and revitalisation.

China is not unique in having to deal with rural revitalisation. The confusion that China faces regarding rural heritage is a problem that other countries have confronted or are confronting. Accordingly, this special issue aims to create an international platform for dialogue about rural heritage practices. Coming from different disciplines and focusing on sustainable development of rural heritage, experts from different countries discuss methods of rural heritage conservation and revitalisation, policy, and management systems from their various perspectives. The articles in this special issue address specific topics related to rural heritage and do not present a singular, particular viewpoint; however, the articles are inherently connected, and to a certain extent they reveal common dilemmas faced the world over. Thus, it is necessary to consider how we should examine the ideas that these articles wish to express. Before tackling that point, we should first explain our understanding of rural heritage from the following two aspects.

What does rural heritage signify?

There is no clear, consummate definition of 'rural heritage'. However, it is widely recognised that rural heritage involves both the architectural heritage in the countryside and the wider geographic, historical, and cultural 


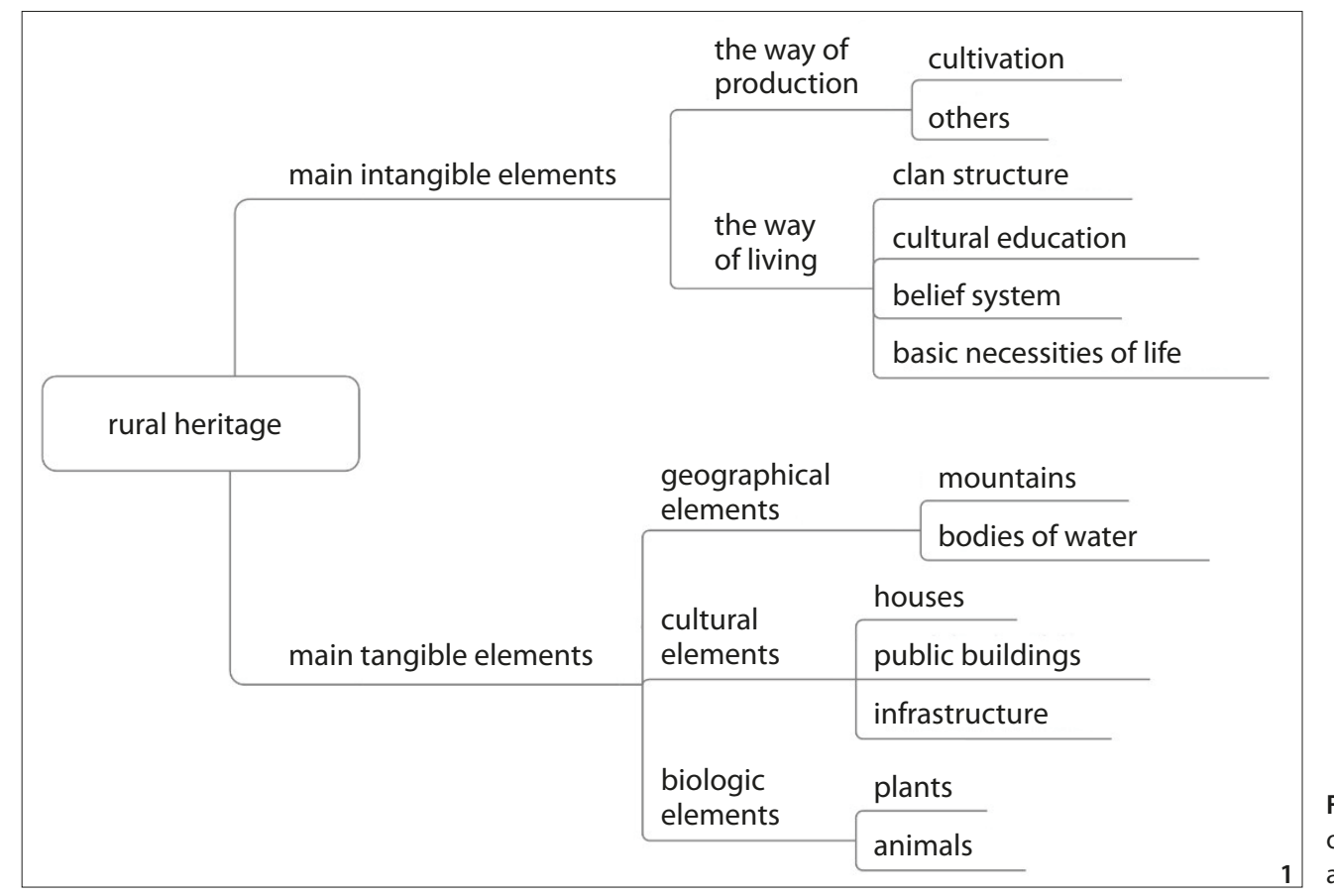

Figure 1 Constituent elements of rural heritage (Source: Shi and Zhao 2018).

background. Considerations of the integrity of rural heritage have developed from the concept of cultural landscape. The notion of cultural landscape originates from that of cultural geography, which began with the geographer Carl Ortwin Sauer in The Morphology of Landscape in 1925. He defined cultural landscape as 'fashioned from a natural landscape by a culture group' and stated, 'Culture is the agent, the natural area is the medium, the cultural landscape the result' (Sauer 1925). Cultural landscape goes beyond the concept of geography: it became a 'prevalent study' in the field of cultural heritage protection after becoming a special category of World Heritage in 1992. Cultural landscape has been gradually recognised and understood worldwide. In the Operational Guidelines for the Implementation of the World Heritage Convention, 'cultural landscape' is defined as 'combined works of nature and of man' (WHC 2017). Therefore, traditional rural areas with a long history are an important object of cultural geography research and also an important part of the World Heritage system (Fowler 2003). Moreover, because rural areas are human living spaces, people, animals, and plants change with time, and the architecture and infrastructure are constantly updated. Therefore, even if rural heritage is managed under a framework of heritage protection, its dynamic attributes will not change (Shi and Du 2019; Liu, Du and Ren 2019).

Why does the World Heritage system consider cultural landscape a special category of cultural heritage? The purpose with the World Heritage system is to express the value of a cultural landscape as a whole: it is based on the recognition that a cultural landscape is a comprehensive framework, which includes all kinds of natural and human elements. Accordingly, cultural landscape can be considered using a holistic methodology; that can be applied to protect the heritage-especially protection of rural areas where nature and culture intermingle. Thus, there are many similar expressions related to rural heritage, such as rural landscape as heritage (ICOMOS 2017; Scazzosi, Wang and Li 2018), agricultural heritage ${ }^{2}$, built heritage $^{1}$, rural cultural landscape (Shi 2013), and village cultural landscape (Du, Hou and Zhao 2018); however, the essence of these concepts is greatly influenced and enlightened by the methodology of cultural landscape (Shi 2016). When we talk about rural heritage, we are not trying to establish a new type of heritage; we are attempting to construct a comprehensive, flexible framework according to the methodology of cultural landscape to examine the characteristics and changes in various tangible and intangible elements as a whole related to rural areas (Figure 1).

What supports the value of rural heritage? What complex elements of rural heritage form an organic system?

The answer to both questions is the human-land relationship. Complex elements constitute the rural heritage as an organic system, which shows extraordinary vitality, resilience, and an inherent sense of place (Figure 2). It is humans who undertake continuous operations from one generation to the next and integrate diverse elements (Shi and Zhao 2018). 


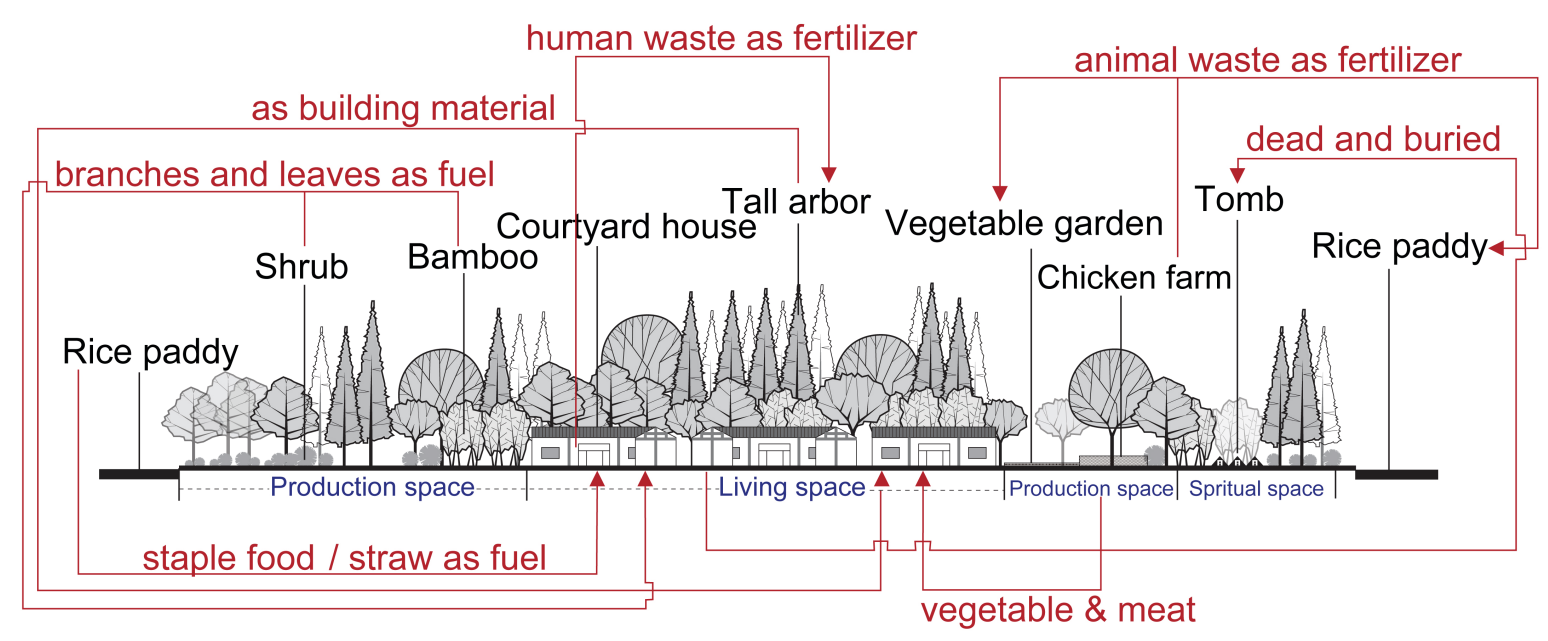

Figure 2 Example of a traditional human-land relationship in villages on the Chengdu Plain, China (Source: Shi 2013)

Thus, rural heritage may be regarded as the crystallisation and outcome of agricultural civilisation. Rural heritage is also a precious type of cultural heritage that developed in the process of civilisation. From the perspective of cultural landscape, rural heritage arises through local residents' unique production methods and lifestyles, which continuously act on the physical environment over a long period of time. Rural heritage is the result of the ongoing interaction between humans and nature: it shows that humans and nature exist in harmony (Han 2007; Shan 2010). The integrated value of rural heritage is borne by diverse tangible and intangible elements; the specific, invisible human-land relationship behind it all is the key to understanding the value of rural heritage. However, rural heritage is also a readable text for studying the humanland relationship in rural areas (Du and Zhao 2018).

A key question has to be asked: in a rural setting, do people and the land interact in a constant manner?

The answer is clearly no. From the perspective of materialist philosophy, movement is eternal: everything has a starting point and will ultimately end. Therefore, recognition of a 'living' rural heritage has to involve the dimension of time in addition to the three spatial dimensions. Put simply, the core of change in the rural heritage lies in the human-land relationship. Before conservation of the rural heritage can be discussed, it is necessary to judge the stages that villages occupy in terms of their development through the characteristics of the human-land relationship. The following questions need to be addressed. What is the traditional human-land relationship of such villages? Are the villages in danger of ceasing to exist? What is the state of the new human-land relationship? Can the villages exist in their present form?
Following a large-scale field survey of Chinese rural heritage conducted by the Centre for Land and Cultural Resources Research at Fudan University in recent years (Liu et al. 2018), at least three typical kinds of rural heritage could be identified. First, the traditional relationship between humans and the land was healthy and could be sustained: the villages were still able to obtain substantial benefits by relying on traditional agriculture, forestry, animal husbandry, sidelines, and fishery. The villages may not have been able to retain all their traditional buildings, but reconstructed traditional buildings could meet the modernlifestyle needs of young people. Second, the traditional relationship between people and the land tended to decline. As the most important means of production, the contribution of land to the villagers' income showed a decrease. Such villages were normally at considerable distance from urban areas; with the outflow of residents, the vacancy rate of buildings began to increase. Even if many traditional buildings could be retained, the overall decline was unavoidable. Third, a new type of human-land relationship became established: it was by traditional buildings being basically retained but with new relationships established through new types of industry. The sustainability of that new type of relationship needs to be tested over time. However, it can be said that the above three characteristic types are somewhat extreme: rural heritage typically shows the coexistence of multiple characteristic states (Shi and Du 2019).

The changes in the human-land relationship in Chinese villages can be compared with the situation in Japan. According to Nobu Kuroda in this special issue, villages in Japan can also be roughly divided into three categories: ones that can maintain traditional farming; ones that will gradually disappear naturally; and ones that need to 
be transformed into cultural heritage sites through planning and design. In our opinion, the nature of this type of 'conservation design' is to reconstruct the human-land relationship in rural areas. Kuroda takes as examples villages in Shirakawa-go and Gokayama (famous Japanese World Heritage Sites) to discuss the constituent elements, authenticity, and basics of changes with the rural heritage; she stresses that the human element is the core in rural heritage protection and that rural revitalisation must respect the will of the local community. Against the background of disappearance of the traditional human-land relationship, villagers are no longer dependent on farming for their main income but on tourism; thus, a new human-land relationship has been established there.

Rural tourism does, however, seem to be an effective means of constructing a new human-land relationship in rural heritage. Like agriculture and handicrafts, tourism can also be regarded as a rural industry. Tourism can use both local and external support in maintaining the vitality of the rural heritage. Similar to Japan, France is rich in rural heritage resources, and rural areas in France have experienced difficulties in the early stages of urbanisation. As to how France uses tourism to revitalise its countryside, Alain Bourdin, Tingting Wan and Philippe Delbos in this special issue find that rural tourism and heritage can exist in a virtuous cycle. They examine the interesting example of the department of Gers in France. The authors analyse the policy of restoration and development in promoting and preserving the heritage, and they conclude that the method has proved effective. They emphasise the importance of coordination, which is a key element in the human-land relationship. The various actions undertaken by local, regional, and department authorities are very impressive: they provide a valuable guide for rural heritage management.

Thus, it is evident that architectural changes in rural areas are actually through the establishment of new human-land relationships. Architecture alone cannot carry the full value of rural heritage; however, architectural design is an indispensable element in improving the quality of that heritage. The planning and design of rural architecture is critical for the cultural landscape, identity, and sense of place with the rural heritage. As a neighbour of France, Germany has a rural building culture that was once neglected. In their article, Xiaoping Xie and Tobias Krüger indicate how the development of building culture can be promoted in rural parts of Germany; they present various methods that have been implemented in some villages as examples of the best type of practice. The experience in Germany demonstrates that revitalisation of building culture is an effective measure of rural revitalisation. The efforts undertaken by France and Germany have considerable relevance for conservation and revitalisation of China's rural heritage.

It is important to consider also the intangible elements in the rural heritage. The sense of place in the rural landscape is the result of the human-land relationship. Ken Taylor analyses the cases of Miao and Dong villages in southwest China. He finds that against the background of tourism development, sustainable development, and poverty eradication, the cultural landscape there has changed under the influence of heritage management policy. Some scholars doubt whether tourism has a negative impact on rural heritage: dance and music performances or other activities for visitors are outside the traditional situation and not authentic. Thus, we might question the accuracy of using the term 'sense of place' in such situations. Taylor believes it is necessary to respect the views of locals when evaluating whether tourism has had a damaging or negative influence in a particular place. Policy makers should take local people's perspectives into account. Taylor believes that even without any interference, tradition will eventually change. Culture and cultural values change in time and space; tourism does not always do harm to a place-based sense of identity or render it flat and inauthentic (Oakes 1997). It can be said that Taylor draws this conclusion based on changes in the humanland relationship in a small part of southwest China. His inference may appear harsh to heritage conservation workers who wish to maintain the status quo. Taylor's study is a reminder that rural heritage is a four-dimensional structure. Under the premise that the human-land relationship has undergone fundamental changes, no one can halt changes in the constituent elements-whether material or immaterial-of the rural heritage. Thus, it is necessary to examine what to protect and to what extent.

At present in China, it is not only ethnic minority villages in the country's southwest that are facing the dilemma of conservation and development. In the developed eastern coastal areas and other rural areas, the impact of modernisation began much earlier and had a deeper impact: through better transport infrastructure, changes in traditional human-land relationship were often faster there. Accordingly, over the past two decades, to promote conservation of rural heritage from state to local authority, government departments at all levels have issued a series of protection measures. Thus, the existing policy system in China relating to rural heritage conservation and rural 
revitalisation is very complex. In her article, Anna-Paola Pola makes a detailed analysis of China's rural policy system: she presents a complete picture of the Chinese approach to rural heritage conservation. In doing so, she offers an excellent guide for readers unfamiliar with China's rural heritage protection and development system.

The rural heritage protection system in China did not develop overnight and at present is far from perfect. But it has at least gradually developed and improved alongside an understanding of rural heritage. Within that huge system, the Historical and Cultural Villages as well as the Traditional Villages constitute the most two important policy areas. Those two related, though different, systems, were also established in the order of time. In their article, Mengyuan Zhou, Songfeng Chu, and Xiaofan Du use an evaluation index system to explain why the protection system of Traditional Villages is more progressive than that of Historical and Cultural Villages. From an investigation of five provinces with traditional villages in 2018 (Liu et al. 2018), Zhou, Chu, and Du found that the economic support for such communities is no longer agriculture: it is clear that without new industries, such villages will become impoverished or cease to exist. For governments at all levels, it is necessary to improve the economy in traditional villages; however, providing a rural heritage with the status of Traditional Village and providing certain financial subsidies is insufficient support. For a traditional village, it may appear that the only way to avoid its economic predicament is by developing tourism. The economic situation, culture, and geographic environment of traditional villages differ greatly, and so it is not possible for them all to adopt the same approach. However, the concept of the eco-museum examined by Zhou, Chu, and $\mathrm{Du}$ is an area of particular interest, and it could have important repercussions for the future of China's rural heritage. In light of the predicament in which traditional villages find themselves and based on a considerable amount of field research, the authors offer three suggestions at the end of their paper toward rebuilding the human-land relationship.

From an analysis of the contributions to this special issue, there is evidently consensus about the relationship between conservation and revitalisation: without conservation, revitalisation has no foundation; without revitalisation of rural heritage, conservation is impossible; thus, the two cannot be separated. At the same time, local residents constitute the core of rural heritage conservation: they are the main body of constructing, maintaining, and developing the human-land relationship in rural heritage; without their subjective initiative, conservation and revitalisation of the rural heritage is impossible.

Further, it is clear from this discussion of the six articles that although the issues faced by various countries and regions are dissimilar, they are all on the way to rebuilding a healthy, harmonious human-land relationship in rural areas. Because of the dynamic characteristics of rural heritage, it is necessary to guide and maintain it toward ensuring its future development in a positive fashion. Accordingly, rural heritage has to be considered seriously in both developed and developing regions. From the perspective of reconstructing the human-land relationship, it is possible to examine changes to the rural heritage and find the nature through phenomenon. In that way, it will be possible to determine the characteristics of rural heritage and establish appropriate development goals.

Rural heritage represents a kind of cornerstone for the human future. As we attempt to conserve and revitalise rural heritage, we become a community with a shared destiny. By discussing the various issues, it will not be possible to find solutions to them all, but at least we are firmly on the right track. As the Chinese saying goes, the stone of other mountains can be used to attack jade, and the exchange of experiences from various countries and regions is particularly important in this regard. We believe that if you read each article in this special issue carefully from the perspective of the human-land relationship, you will find the effort both enlightening and inspiring.

\section{Notes}

1. http://www.icobhs.com/

2. http://www.fao.org/giahs/en/

\section{References}

Du, Xiaofan, and Xiaomei Zhao, eds. 2018. Xiangcun yichan de hexin yanjiu jiazhi: yi Guizhou Loushang Cun weili [The Research on Core Value of Rural Heritage-take Loushang Village as an Example]. Chengdu: Ba Shu Book Company, Ltd.

Du, Xiaofan, Shi Hou, and Xiaomei Zhao. 2018. “The Protection and Development of Guizhou's Rural Heritage: A Case Study of Loushang Village.” [Guizhou xiangcun yichan de baohu yu fazhan-yi Loushang cun weili.] Journal of Guizhou Minzu University (Philosophy and Social Sciences), no. 3: 57-72.

Fowler, Peter, ed. 2003. World Heritage Cultural Landscapes 1992-2002. Paris: UNESCO.

Han, Feng. 2007. "World Heritage Cultural Landscapes 
and New International Trends." [Shijie yichan wenhua jingguan jiqi guoji xin dongxiang] Chinese Landscape Architecture 23 (11): 18-21.

ICOMOS 2017. “Principles Concerning Rural Landscapes as Heritage' ICOMOS-IFLA.” Accessed 10 January 2019. https://www.icomos.org/images/ DOCUMENTS/Charters/GA2017_6-3-1_RuralLandscapesPrinciples_EN_adopted-15122017.pdf

Liu, Shaoyuan, Songfeng Chu, Shi Hou, Ding Shi, Xiaomei Zhao, Ziyu Qi, Mengyuan Zhou, and Xiaofan Du. 2018. "How to Regenerate Traditional Villages." [Chuantong cunluo ruhe zaisheng] Insight China, no. 30:70-73.

Liu, Shaoyuan, Xiaofan Du, and Hexin Ren. 2019. "Recognition on Rural Heritage." China Cultural Relics News 03-16(006).

Oakes, Tim. 1997. "Ethnic Tourism in Rural Guizhou: Sense of Place and the Commerce of Authenticity." In Tourism, Ethnicity and the State in Asia and Pacific Societies, edited by Michel Picard and Robert Wood, 35-70. Honolulu: University of Hawaii Press.

Sauer, Carl Ortwin. 1925. “The Morphology of Landscape." University of California Publications in Geography 2 (2): 19-53.

Scazzosi, Lionella, Xi Wang, and Jingyu Li. 2018. "Contexts and Concept of Principles Concerning Rural Landscape as Heritage ICOMOS 2017.” [Guoji guji yizhi lishihui guanyu xiangcun jingguan yichan de zhunze (2017) chansheng de yujing yu gainian jiedu] Chinese Landscape Architecture 34 (11): 5-9.

Shan, Jixiang. 2010. Zoujin wenhua jingguan yichan de shijie [Towards the World of Cultural Landscape Heritage]. Tianjin: Tianjin Press.

Shi, Ding, and Dianhong, Zhao. 2018. "Research on Human-land Relationship and Landscape Change of Loushang Village in Shiqian, Guizhou from Cultural Landscape Perspective." [Jiyu wenhua jingguan lilun de Guizhou Shiqian Loushangcun rendi guanxi ji jingguan bianqian yanjiu.] China Cultural Heirtage, no. 2: 31-44.

Shi, Ding, and Xiaofan Du. 2019. "The Type and Characters of Rural Heritage." [Xiangcun yichan de leixing yu tezheng] China Cultural Relics News 03-16(006).

Shi, Ding. 2013. "Research on the Rural Cultural Landscape in the Metropolitan Area of Chengdu, Chinaa Case in Dujiangyan City." PhD diss., University of Tokyo.

Shi, Ding. 2016. "Cultural Landscape Conservation in China Today." Landscape Research Japan: Journal of the Japanese Institute of Landscape Architecture 80 (1): 59-60.

WHC (World Heritage Centre). 2017. Operational Guidelines for the Implementation of the World Heritage Convention. Paris: UNESCO World Heritage Centre. 